\title{
Polydatin regulates proliferation, apoptosis and autophagy in multiple myeloma cells through mTOR/p70s6k pathway
}

This article was published in the following Dove Press journal:

OncoTargets and Therapy

16 February 2017

Number of times this article has been viewed

\author{
Baojun Yang' \\ Shunxin Zhao ${ }^{2}$ \\ 'Department of Pathology, General \\ Hospital of PINGMEISHENMA \\ Medical Group, Pingdingshan, \\ ${ }^{2}$ Department of Respiratory Medicine, \\ The First Affiliated Hospital of \\ Zhengzhou University, Zhengzhou, \\ People's Republic of China
}

Background: Polydatin (PD) plays an important role in suppressing platelet aggregation, reducing blood lipid, restoring microcirculation and protecting from myocardial ischemia/reperfusion injury and shock. In addition, PD possesses anticancer activity. However, the effect and the mechanism of PD in regulating multiple myeloma (MM) cell survival and death are still unknown.

Methods: Cell proliferation and apoptosis of RPMI 8226 cells, respectively, were analyzed by cell counting kit8 (CCK-8) assay and flow cytometry. The levels of caspase-3, cleaved caspase-3, caspase-9, cleaved caspase-9, Bcl-2 and Bax were analyzed by Western blot. Autophagy induced by PD was investigated by detecting the levels of Beclin 1, Atg5, LC3I, LC3II, HSP70 and HSP27. The autophagy inhibitor 3-methyladenine (3-MA), mTOR/p70s6k inhibitor rapamycin, and mTOR activator MHY1485 were used to analyze the mechanism of cell proliferation, apoptosis and autophagy influenced by PD. The phosphorylations of mTOR and p70s6k were detected by Western blot.

Results: A gradual decrease in cell proliferation of RPMI 8226 cells was observed with an increase in PD concentrations $(P<0.05)$. PD also induced cell apoptosis and autophagy in a concentration-dependent manner. Both 3-MA and MHY1485 reversed the inhibitory effect of PD on cell proliferation and attenuated the positive effect of PD on cell apoptosis and autophagy. The phosphorylation of mTOR and p70s6k was significantly suppressed by PD $(P<0.05)$. Furthermore, inhibition of the mTOR/p70s6k signaling pathway by rapamycin significantly induced autophagy and apoptosis and inhibited cell viability $(P<0.05)$.

Conclusion: PD effectively suppressed cell proliferation and induced apoptosis and autophagy of MM cells via the mTOR/p70s6k signaling pathway in a concentration-dependent manner in vitro, indicating that PD could be a potential anticancer drug for MM therapy.

Keywords: polydatin, proliferation, apoptosis, autophagy, multiple myeloma, mTOR/p70s6k

\section{Introduction}

Multiple myeloma (MM), the second most common devastating clonal plasma cell malignancy, occurs in bone marrow with a 45\% 5-year survival rate; its characteristics include uncontrolled proliferation, complex chromosomal instability and accumulation of plasma cells. ${ }^{1,2} \mathrm{MM}$ accounts for $1 \%$ of all cancers and $1 \%-10 \%$ of all clonal plasma cell malignancies. ${ }^{3}$ In addition, more than 40,000 cases are being diagnosed in Europe, and the annual incidence of MM is increasing progressively with age. ${ }^{4}$ Though a number of novel pharmacological strategies have been developed in the past 30 years, including the introduction of new immunomodulatory drugs and proteasome inhibitors, MM still remains an incurable disease. ${ }^{5}$ Thus, new drugs with increased therapeutic efficacy to treat MM are of greater demand.
Correspondence: Baojun Yang

Department of Pathology, General Hospital of PINGMEISHENMA Medical

Group, No I Kuanggong Middle

Road, Xinhua, Pingdingshan 467099,

People's Republic of China

Tel +86375 2799220

Email bandjyang@163.com hereby accept the Terms. Non-commercial uses of the work are permitted without any furcther permission from Dove Medical Press Limited, provided the work is properly attributed. For permission for commercial use of this work, please see paragraphs 4.2 and 5 of our Terms (https://www.dovepress.com/terms.php).
her 
3,4,5'-trihydroxystibene-3-monoglucoside (polydatin, PD), a monocrystalline drug and a glycoside form of resveratrol used in traditional Chinese therapy, is one of the key effective components found in the root and rhizome of herb Polygonum cuspidatum. ${ }^{6}$ Previous pharmacological research and clinical application manifested that PD was mainly used in suppressing platelet aggregation, reducing blood lipid, restoring microcirculation and protecting from myocardial ischemia/reperfusion injury and shock..$^{6-9} \mathrm{PD}$ also possesses anticancer activity. For instance, Wang et al showed that PD effectively suppressed cell proliferation and induced cell cycle arrest in S phase and apoptosis of acute monocytic leukemia. ${ }^{10} \mathrm{Xu}$ et al reported that $\mathrm{PD}$ inhibited cell growth by suppressing $\beta$-catenin signaling pathway and promoted apoptosis by raising the ratio of $\mathrm{Bax} / \mathrm{Bcl}-2$ in human osteosarcoma cells. ${ }^{11}$ Zhang et al found that PD showed a significant proliferation inhibition, dose-dependent apoptosis induction and cell cycle arrest in lung cancer. ${ }^{12}$ However, the effect of PD on MM has not been elucidated.

Mammalian target of rapamycin (mTOR) is located at the intersection of different signal pathways, including Ras, phosphoinositide-3 kinase (PI3K)/protein kinase B (AKT) and nuclear factor-kappa B, toward mRNA, ribosome and protein synthesis. ${ }^{13}$ It was reported that mTOR gets activated and plays an important role in the cell proliferation and growth of tumor cells when its downstream target ribosomal p70S6 kinase (p70s6k) is phosphorylated. ${ }^{14,15}$ In mammalian cells, mTOR/p70s6k signaling pathway is also associated with autophagy when cells encounter conditions such as tumor suppression, oxidative stress and infection. ${ }^{16}$ Besides, activation of the mTOR/p70s6k signaling pathway plays a crucial role in tumorigenesis, angiogenesis and tumor proliferation. ${ }^{17-19}$ Pang et al revealed that celastrol inhibited cell growth and angiogenesis in prostate cancer by targeting the AKT/mTOR/p70s6k signaling pathway induced by vascular endothelial growth factor. ${ }^{20}$ However, the interrelation between PD and mTOR/p70s6k signaling pathway remains unclear. The aim of this study was to investigate the effect of PD on MM cells and its underlying molecular mechanism.

\section{Materials and methods \\ Cell line and culture}

Human MM cell line RPMI 8226 was purchased from American Type Culture Collection (Rockville, MD, USA) and cultured in medium RPMI 1640 (Gibco, Grand Island, NY, USA) supplemented with $10 \%$ inactive fetal bovine serum (Hyclone, Logan, UT, USA) and 1\% penicillin/ streptomycin solution (Thermo Fisher Scientific, Shanghai, People's Republic of China) at $37^{\circ} \mathrm{C}$ under a humidified atmosphere of $5 \% \mathrm{CO}_{2}$ and $95 \%$ air. The cells used for subsequent experiments were obtained from logarithmic phase.

\section{Cell viability assay}

Cell viability was detected by Cell Counting Kit 8 (CCK-8; Beyotime, Shanghai, People's Republic of China). In short, cells were plated into 96-well plates at a concentration of $5 \times 10^{3}$ cells/well and treated with different concentrations $(0,5,10,25,50,100,200$ and $400 \mu \mathrm{mol} / \mathrm{L}$ ) of PD (Nanjing Debjochem Co. Ltd, Nanjing, People's Republic of China) or different concentrations ( 0,100 and 1,000 nmol/L) of rapamycin (Sigma-Aldrich, St Louis, MO, USA). After $24 \mathrm{~h}$ or $48 \mathrm{~h}$ of treatment, $10 \mu \mathrm{L}$ of CCK-8 solution was added to each well and further incubated for $2 \mathrm{~h}$. Then the absorbance values were detected at a wavelength of $450 \mathrm{~nm}$ using a Bio-Rad enzyme-linked immunosorbent assay (ELISA) microplate reader (Bio-Tech Instruments Inc., Winooski, VT, USA). The cell viability was calculated by the optical density (OD) values of treated groups/OD values of control groups $\times 100 \%$.

\section{Assessment of apoptosis}

RPMI 8226 cells were treated with various concentrations $(0,50,100$ and $200 \mu \mathrm{mol} / \mathrm{L})$ of $\mathrm{PD}$, different concentrations $(0,100$ and $1,000 \mathrm{nmol} / \mathrm{L})$ of rapamycin, or PD $(200 \mu \mathrm{mol} / \mathrm{L})$ together with autophagy inhibitor 3-MA $(10 \mathrm{mmol} / \mathrm{L})$, respectively. After $24 \mathrm{~h}$ of treatment, Annexin $\mathrm{V}$-fluorescein isothiocyanate (FITC) Apoptosis Detection Kit (Beyotime) was used to analyze the percentage of apoptotic cells. Briefly, cells were collected, trypsinized and washed with phosphate-buffered saline (PBS). Then, the cells were mixed with binding buffer to a final concentration of $3 \times 10^{5}$ cells per well, stained with Annexin V-FITC-propidium iodide (PI) and incubated for $10 \mathrm{~min}$ at room temperature in the dark. Stained cells were subjected to FACSCalibur flow cytometer (BD Biosciences, San Jose, CA, USA).

\section{Western blot analysis}

After the RPMI 8226 cells were collected and lysed, the concentration of proteins was determined by bicinchoninic acid Protein Assay Kit (CoWin Biotechnology, Beijing, People's Republic of China). Equal amount of proteins were loaded on a $10 \%$ sodium dodecyl sulfate-polyacrylamide gel electrophoresis (SDS-PAGE) and then blotted to polyvinylidene fluoride membranes. The membranes were blocked with $1 \times$ PBS containing 5\% skimmed milk 
and $0.1 \%$ Tween-20 and then incubated with desired primary antibodies (anti-Bcl-2, anti-Bax, anti-caspase-3, anti-cleaved caspase-3, anti-caspase-9, anti-cleaved caspase-9, anti-Beclin 1, anti-Atg5, anti-LC3I, anti-LC3II, anti-heat shock protein (HSP)70, anti-HSP27, anti-t-mTOR,

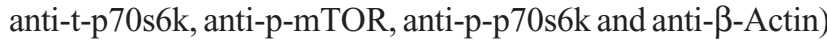
(Santa Cruz Biotechnology, Santa Cruz, CA, USA) overnight at $4^{\circ} \mathrm{C}$. After washing three times with PBS, the membranes were immunoblotted with appropriate secondary antibodies and incubated for $1 \mathrm{~h}$ at room temperature. The immunoreactive bands of specific proteins were visualized by enhanced chemiluminescence (Santa Cruz Biotechnology) and analyzed using an Image Scanner (Amersham Biosciences, Uppsala, Sweden). $\beta$-actin was used as an internal control.

\section{Statistical analysis}

Data from each group were expressed as mean \pm standard deviation (SD) or mean \pm standard error. SPSS19.0 software package (SPSS Inc., Chicago, IL, USA) was used to analyze all statistics. Differences between two groups were performed using the Student's $t$-test and differences among multiple groups were analyzed by using one-way analysis of variance. $P<0.05$ was considered to indicate a statistically significant difference.

\section{Results}

\section{PD suppressed proliferation of RPMI 8226 cells}

CCK-8 assay was performed to investigate the effect of PD on cell viability after RPMI 8226 cells were treated with a concentration gradient of PD for $24 \mathrm{~h}$ and $48 \mathrm{~h}$. The results showed that the percentage of cell viability significantly decreased along with an increase in PD concentration at $24 \mathrm{~h}$ and $48 \mathrm{~h}$, indicating that PD effectively inhibited cell viability in time- and concentration-dependent manners (Figure 1). Moreover, our results demonstrated that PD showed cytotoxicity with $\mathrm{IC}_{50}$ values of $131 \mu \mathrm{M}$ and $93 \mu \mathrm{M}$ in RPMI 8226 cells at $24 \mathrm{~h}$ and $48 \mathrm{~h}$, respectively.

\section{PD induced apoptosis of RPMI 8226 cells}

To further assess the cytotoxicity of PD on RPMI 8226 cells, the cells were treated with various concentrations $(0,50,100$ and $200 \mu \mathrm{mol} / \mathrm{L})$ of PD for $24 \mathrm{~h}$. Subsequently, the cells were double stained by Annexin V-FITC/PI and apoptosis was assessed by flow cytometry. The results obtained demonstrated that the apoptosis rates dramatically increased in a concentration-dependent manner (Figure 2A). Besides, apoptosis indicator, such as cleaved caspase-3, cleaved caspase- 9 and proapoptotic protein Bax, were upregulated in a concentration-dependent manner, while antiapoptotic protein Bcl-2 was downregulated with increased concentrations of PD treatment (Figure 2B). These results suggest that PD contributed to the induction of apoptosis.

\section{PD induced autophagy in RPMI 8226 cells}

To further study the effect of PD on autophagy, the levels of autophagy markers LC3I and LC3II and autophagy-related proteins Atg5 and Beclin 1 were detected by Western blot. As shown in Figure 3A and B, PD significantly increased the expression of Beclin 1, Atg5 and LC3II. Moreover, an augmentation in the conversion of cytosolic form of LC3 (LC3I) into active membrane-bound form of LC3 (LC3II)

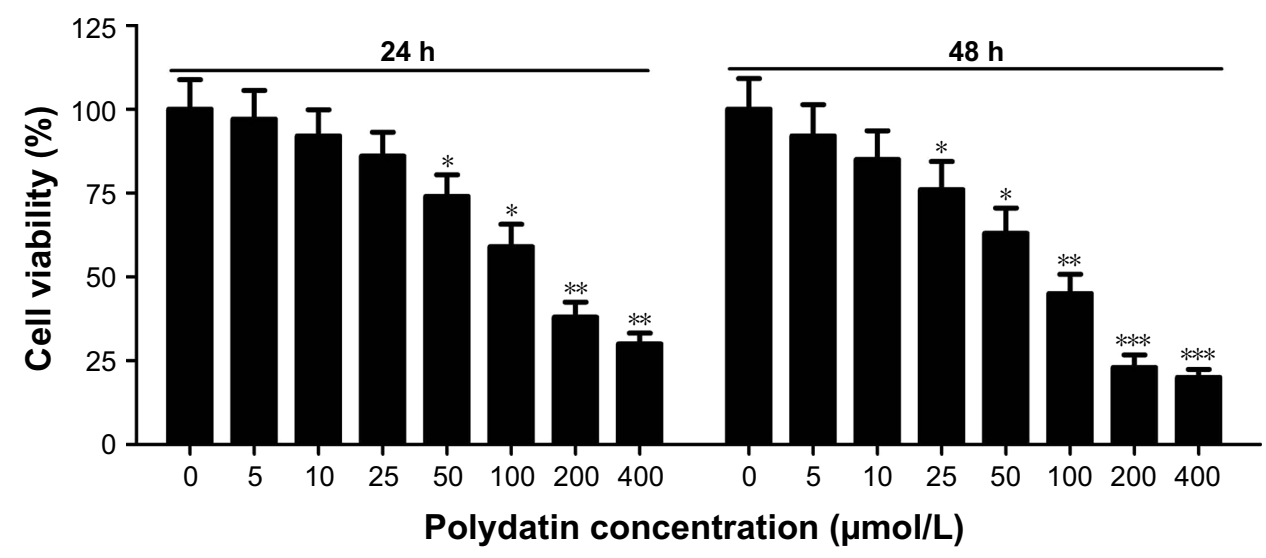

Figure I Effect of PD on proliferation in RPMI 8226 cells. CCK-8 assay was used to examine the cell viability after RPMI 8226 cells were treated with PD at different concentrations for $24 \mathrm{~h}$ and $48 \mathrm{~h}$. Each group was analyzed by three independent experiments. Data are presented as mean $\pm S E M$. $* P<0.05$, $* * P<0.0 \mathrm{I}$, $* * * P<0.00 \mathrm{I}, \mathrm{compared}$ to $0 \mu \mathrm{mol} / \mathrm{L}$.

Abbreviations: SEM, standard error of the mean; PD, polydatin; CCK-8, cell counting kit8. 
A

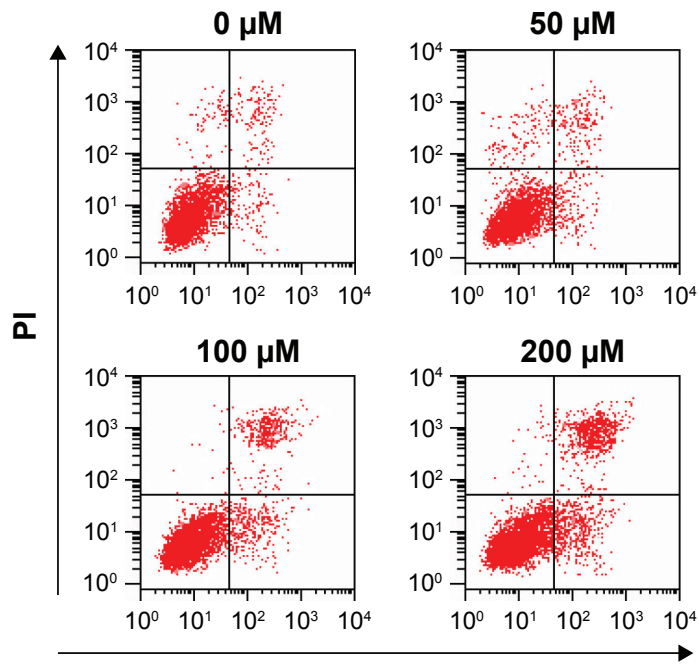

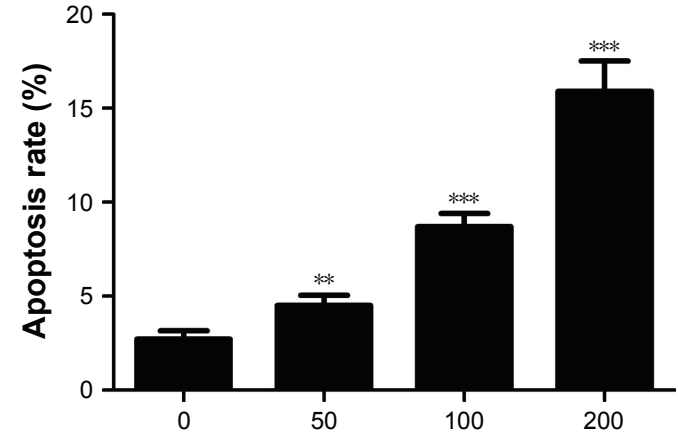

Polydatin concentration ( $\mu \mathrm{mol} / \mathrm{L})$

Annexin V-FITC

$\mathbf{B}$

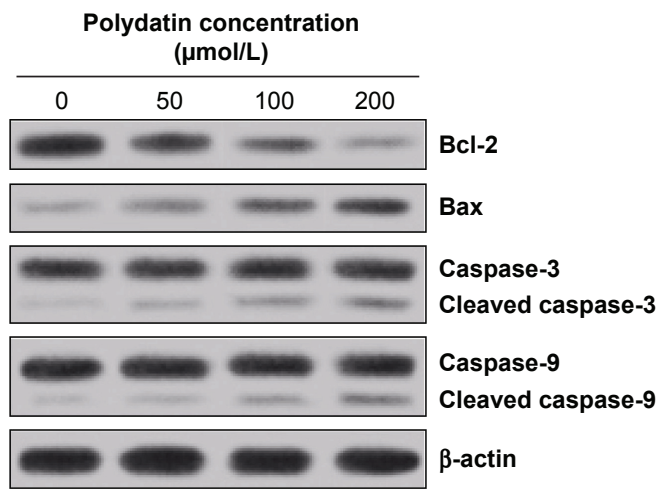

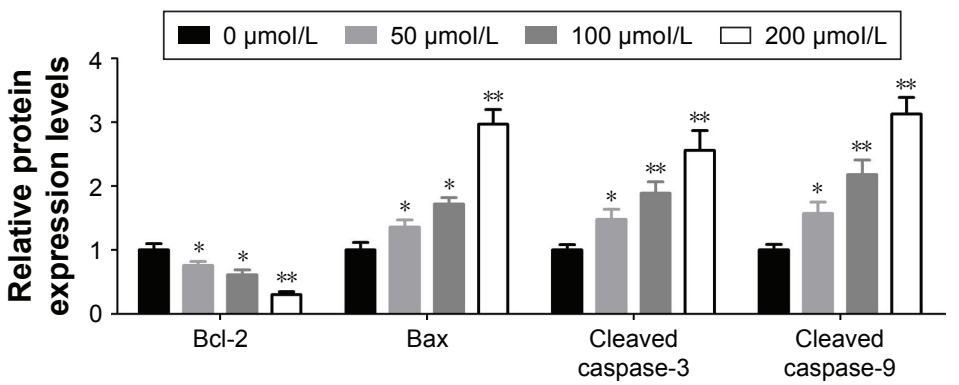

Figure 2 PD induced apoptosis of RPMI 8226 cells. (A) Apoptosis rates with different concentrations (0, 50, 100 and $200 \mu$ mol/L) of PD treatment were assessed by flow cytometry followed by Annexin V-FITC/PI staining. (B) The levels of apoptosis-associated proteins cleaved caspase-3, cleaved caspase-9, Bcl-2 and Bax in RPMI 8226 cells treated with various concentrations $(0,50,100$ and $200 \mu \mathrm{mol} / \mathrm{L})$ of PD were detected by Western blot and normalized to $\beta$-actin. Each group was analyzed by three independent experiments. Data are presented as mean $\pm S D$. $* P<0.05$, $* * P<0.0 \mathrm{I}, * * * P<0.00 \mathrm{I}$ compared to $0 \mu \mathrm{mol} / \mathrm{L}$.

Abbreviations: SD, standard deviation; PD, polydatin; FITC, fluorescein isothiocyanate; PI, propidium iodide.

(ie, the ratio of LC3II/I) was detected in a concentrationdependent manner (Figure 3C). In addition, the relationship between the level of HSP and autophagy was assessed by Western blot and the results revealed that the expressions of HSP27 and HSP70 were significantly decreased when the cells were treated with PD (Figure 3D and E).

\section{3-MA overturned the inhibitory effect of PD on proliferation and attenuated the induced effect of PD on apoptosis and autophagy in RPMI 8226 cells}

The autophagy inhibitor 3-MA was used to further verify the cytotoxicity effect of PD on RPMI 8226 cells. The CCK- 8 assay results showed that the cell viability of RPMI 8226 cells when treated with PD and 3-MA significantly increased compared with PD-treated group (Figure 4A). In addition, 3-MA dramatically decreased the apoptosis rates induced by PD (Figure 4B). 3-MA significantly upregulated Bcl-2 and downregulated Bax compared with PD-treated group (Figure 4C). Meanwhile, 3-MA significantly reduced the positive role of $\mathrm{PD}$ on cleaved caspase-3 and cleaved caspase-9 expression (Figure 4D and E). Moreover, in comparison with the PD-treated group, the levels of Beclin 1, Atg5 and LC3II and the ratio of LC3II/ LC3I both obviously decreased in 3-MA + PD-treated group (Figure 4F-H).

\section{PD suppressed the activation of mTOR/ P70s6k signaling pathway in RPMI 8226 cells}

Since $\mathrm{mTOR} / \mathrm{p} 70 \mathrm{~s} 6 \mathrm{k}$ signaling pathway played a crucial role in regulating apoptosis and autophagy, the effect of $\mathrm{PD}$ on $\mathrm{mTOR} / \mathrm{p} 70 \mathrm{~s} 6 \mathrm{k}$ signaling pathway was investigated. The expression levels of key components in this pathway 
A

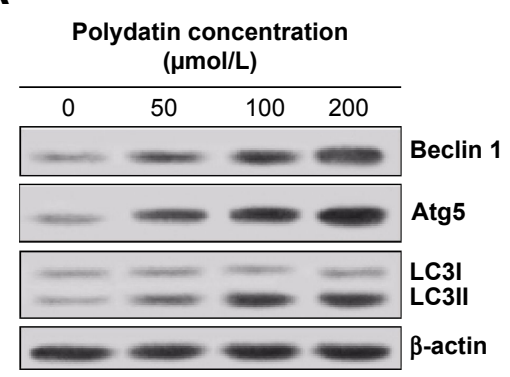

C

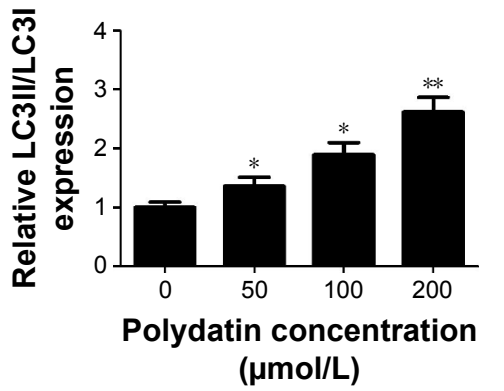

B

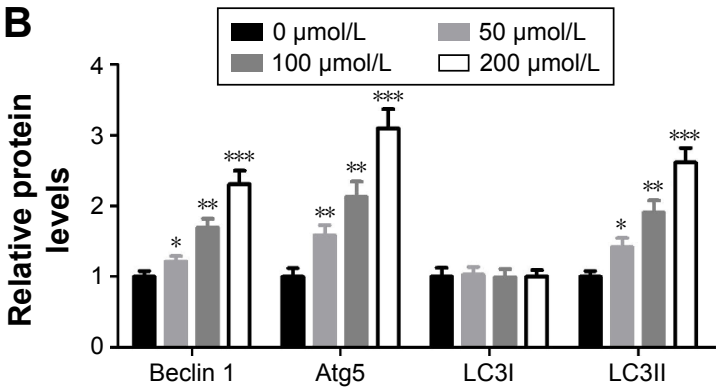

D

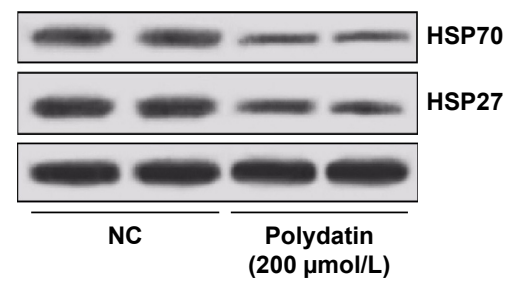

E

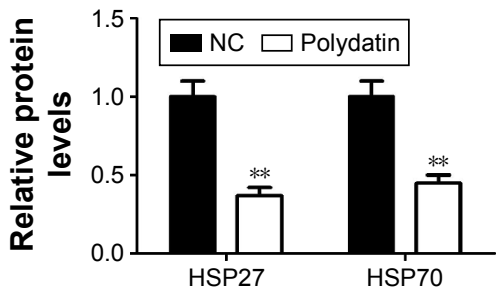

Figure 3 PD induced autophagy in RPMI 8226 cells. (A) Levels of Beclin I, Atg5, LC3I and LC3II were determined by Western blot after cells were treated with different concentrations $(0,50,100$ and $200 \mu \mathrm{mol} / \mathrm{L})$ of PD for $24 \mathrm{~h}$. $\beta$-actin was used as an internal control. (B) Quantification analysis of relative proteins Beclin I, Atg5, LC3I and LC3II in RPMI 8226 cells by Western blot. (C) The ratio of LC3II/I was calculated. (D and E) Western blot analysis of HSP70 and HSP27 in RPMI 8226 cells treated with $200 \mu \mathrm{mol} / \mathrm{L}$ PD for $24 \mathrm{~h}$. Each group was analyzed by three independent experiments. Data are presented as mean \pm SD. $* P<0.05$, $* * P<0.0 \mathrm{I}$, $* * * P<0.00 \mathrm{I}$, compared to $0 \mu \mathrm{mol} / \mathrm{L}$.

Abbreviations: HSP, heat shock protein; SD, standard deviation; PD, polydatin; NC, negative control.

were examined by Western blot. As shown in Figure $5 \mathrm{~A}$ and $\mathrm{B}, \mathrm{PD}$ effectively decreased the phosphorylation of mTOR and p70s6k compared with the control, indicating that PD could effectively suppress mTOR/p70s6k signaling pathway.

\section{Rapamycin inhibited viability and induced apoptosis and autophagy in a concentration-dependent manner in RPMI 8226 cells}

Rapamycin, a specific inhibitor of mTOR/p70s6k signaling pathway, was used to further determine the role of mTOR/p70s6k signaling pathway in MM cells. The results showed that compared with control group, an increased expression of Beclin 1 and Atg5 and conversion of endogenous LC3I into LC3II were observed in rapamycin-treated cells (Figure 6A-C). Meanwhile, rapamycin treatment led to an evident decrease in cell viability (Figure 6D) and an obvious increase in apoptosis rates compared with control group (Figure 6E). Moreover, Bax, caspase-3 and caspase-9 expressions were strikingly elevated and Bal-2 was drastically reduced in cells treated with rapamycin (Figure 6F and G). In summary, pretreatment of rapamycin significantly inhibited viability, promoted apoptosis and induced autophagy in RPMI 8226 cells.

\section{MHYI 485 relieved the inhibitory effect of PD on proliferation and attenuated the induced effect of PD on apoptosis and autophagy in RPMI 8226 cells}

mTOR activator MHY1485 was used to further verify the effect of activation of the mTOR/p70s6k signaling pathway on cytotoxicity of PD in RPMI 8226 cells. CCK-8 assay showed that cell viability of RPMI 8226 was significantly higher in PD + MHY1485 group than that in PD group (Figure 7A). Flow cytometry results indicated that PD markedly promoted apoptosis of RPMI 8226 cells, whereas MHY1485 strikingly attenuated this effect (Figure 7B). Furthermore, Western blot analysis exhibited that PD treatment remarkably improved the levels of Beclin 1, Atg5 and LC3II and the ratio of LC3II/LC3I, which was obviously abated by MHY1485 (Figure 7C). Taken together, these findings revealed that activation of the mTOR/p70s6k signaling pathway conspicuously attenuated the inhibitory effect of PD on proliferation and abrogated the induced effect of PD on apoptosis and autophagy in RPMI 8226 cells. 
A

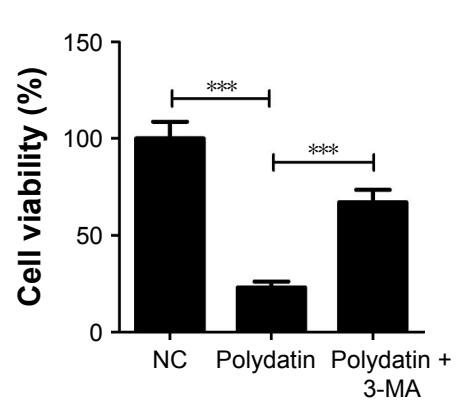

B

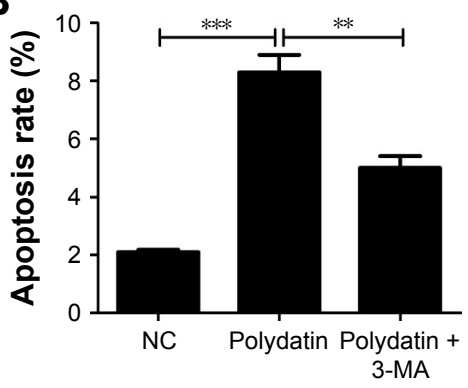

C

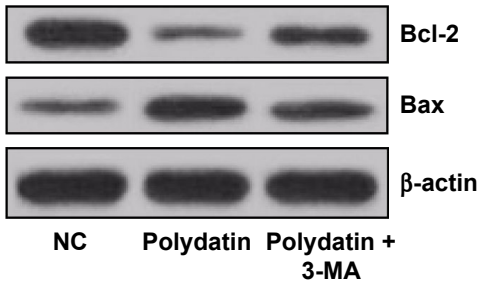

D
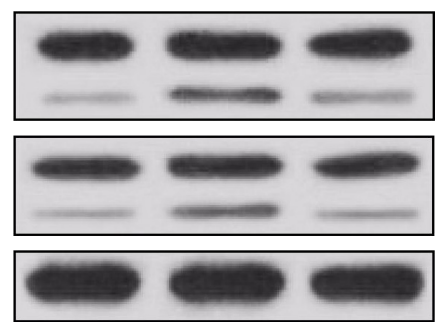

NC

Polydatin $\underset{\text { 3-MA }}{\text { Polydatin }}+$

E

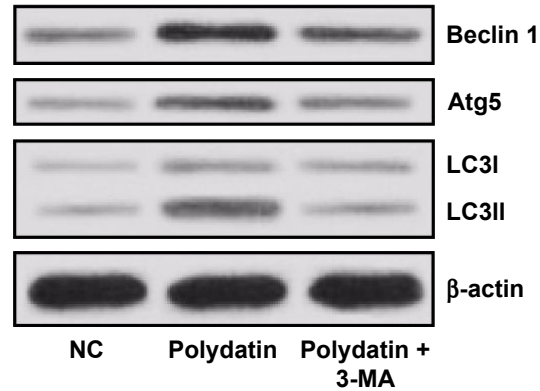

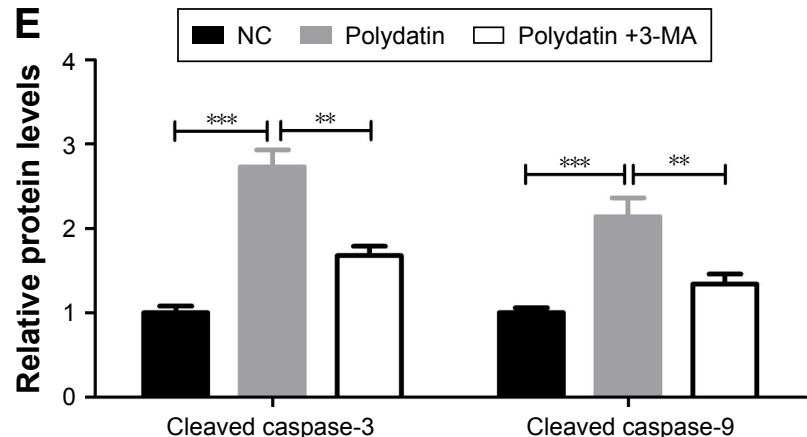

G $\square$ NC $\square$ Polydatin $\square$ Polydatin +3-MA

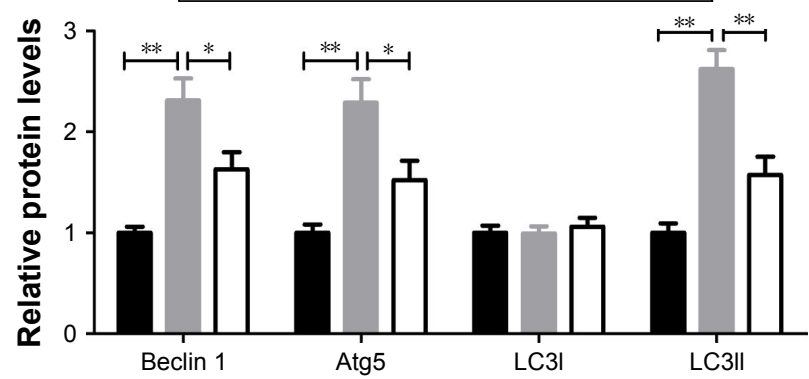

H

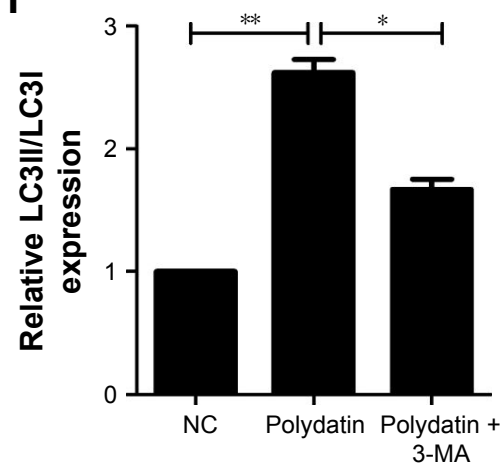

Figure 4 Viability repression, apoptosis and autophagy induced by PD were inhibited by 3-MA in RPMI 8226 cells. Cells were treated with $200 \mu$ mol/L PD alone or in combination with $10 \mathrm{mmol} / \mathrm{L}$ 3-MA for $24 \mathrm{~h}$. (A) Cell viability was assessed by CCK-8 assay. (B) The apoptosis rates of RPMI 8226 cells were determined by flow cytometry. (C and D) Western blot analysis of Bcl-2, Bax, caspase-3, cleaved caspase-3, caspase-9 and cleaved caspase-9. $\beta$-actin was used as a control. (E) Quantification analysis of cleaved caspase- 3 and cleaved caspase- 9 by densitometry. (F) Western blot was used to analyze the expressions of Beclin I, Atg5, LC3I and LC3II. $\beta$-actin was used as a control. (G) Quantification analysis of Beclin I, Atg5, LC3I and LC3II. (H) The ratio of LC3II/ LC3I. Data are presented as mean \pm SD. $* P<0.05$, $* * P<0.0$ I, $* * * P<0.00$ I, compared to control.

Abbreviations: SD, standard deviation; PD, polydatin; NC, negative control; CCK-8, cell counting kit8.

\section{Discussion}

MM treatment has achieved greater development in recent years due to the application of novel efficient agents such as immunomodulatory drugs and proteasome inhibitors.
However, although response rate and overall survival of MM patients have increased, most of the patients appear to relapse or remain refractory to therapy. Thus, it is of high significance to find new therapeutic strategies for MM treatment. 
A

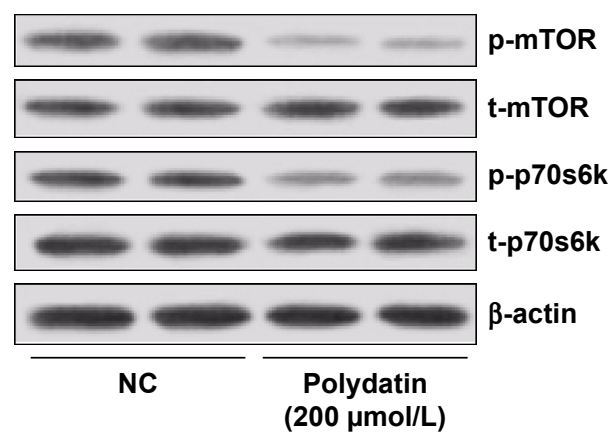

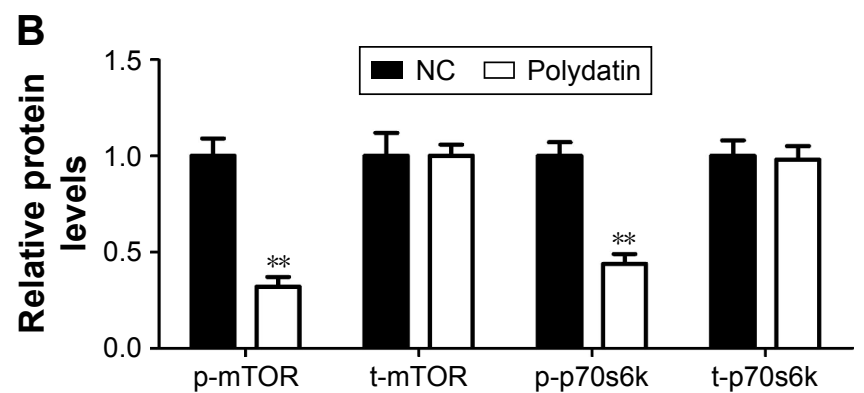

Figure 5 PD suppressed the activation of mTOR/p70s6k signaling pathway in RPMI 8226 cells. (A) Western blot analysis of p-mTOR, t-mTOR, $p-p 70 s 6 k$ and t-p70s6k in RPMI 8226 cells treated with $200 \mu \mathrm{mol} / \mathrm{L}$ PD. (B) Quantification analysis of relative proteins p-mTOR, t-mTOR, p-p70s6k and t-p70s6k. Data are presented as mean \pm SD. $* * \mathrm{P}<0.01$, compared to $\mathrm{NC}$.

Abbreviations: SD, standard deviation; PD, polydatin; NC, negative control; p-mTOR, phosphorylated mammalian target of rapamycin; t-mTOR, total mammalian target of rapamycin.

A

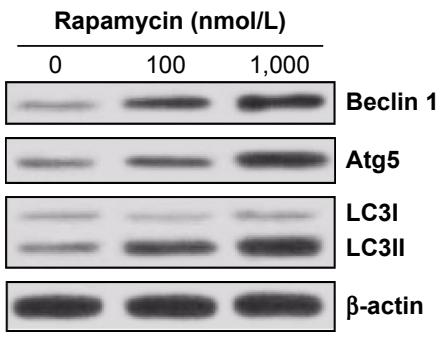

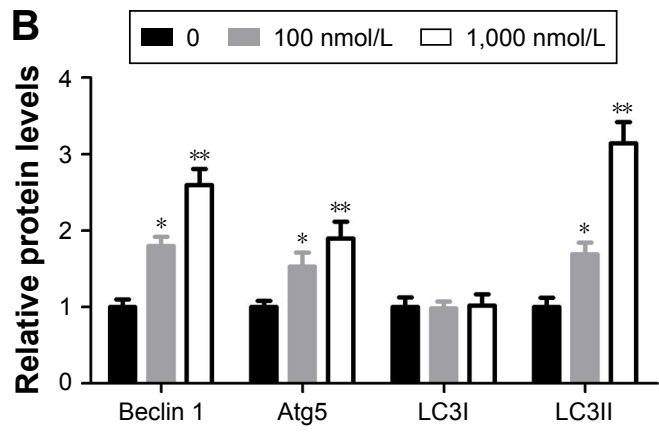

E

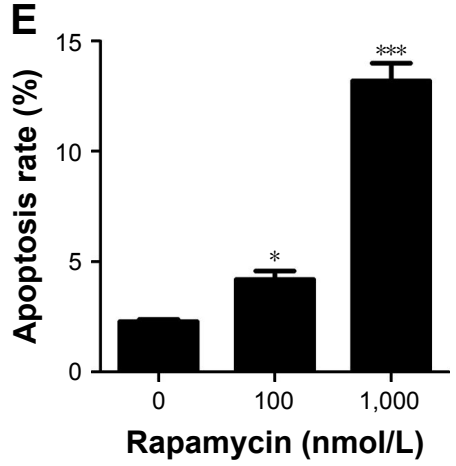

$\mathbf{F}$
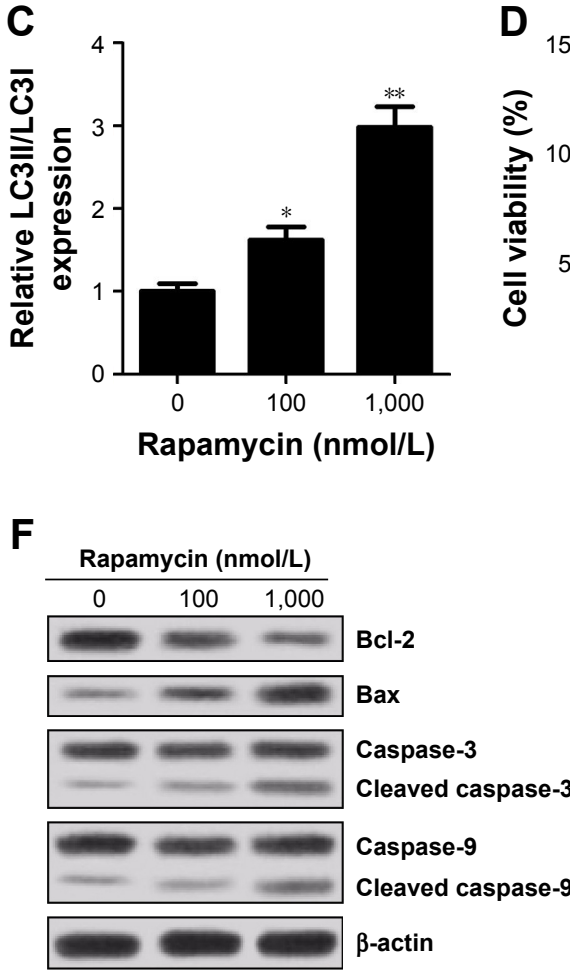
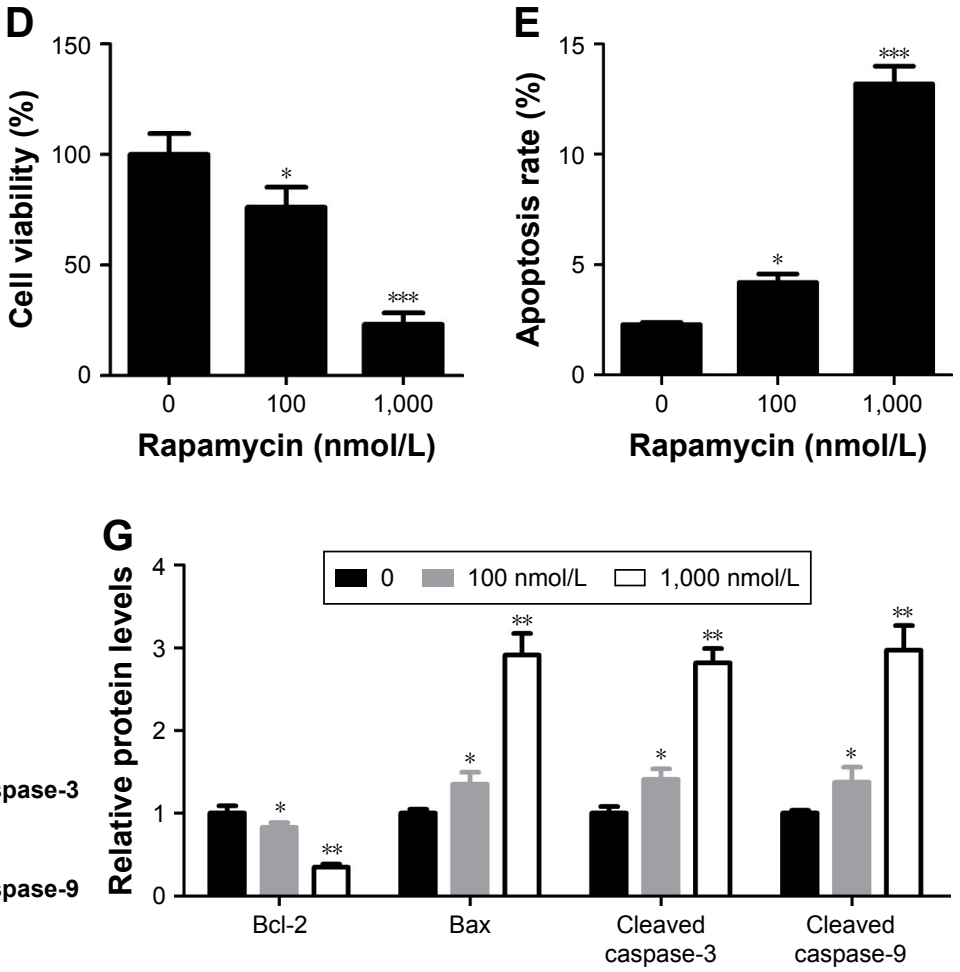

Figure 6 Effect of rapamycin on autophagy, viability and apoptosis of RPMI 8226 cells. Cells were treated with different concentrations (0, I00 and I,000 nmol/L) of rapamycin for 24 h. (A and B) Expressions of Beclin I, Atg5, LC3I and LC3II were determined by Western blot. $\beta$-actin was used as an internal control. (C) The ratio of LC3II/LC3I. (D) Cell viability was determined by CCK-8 kit. (E) Cell apoptosis rates were assessed by flow cytometry. (F and $\mathbf{G})$ Expressions of caspase-3, cleaved caspase-3, caspase-9, cleaved caspase-9, Bcl-2 and Bax were measured by Western blot and normalized to $\beta$-actin. Data are presented as mean \pm SD. $* P<0.05$, $* * P<0.0 \mathrm{I}$, $* * * P<0.00 \mathrm{I}$, compared to $0 \mu \mathrm{mol} / \mathrm{L}$.

Abbreviation: SD, standard deviation; CCK-8, cell counting kit8. 

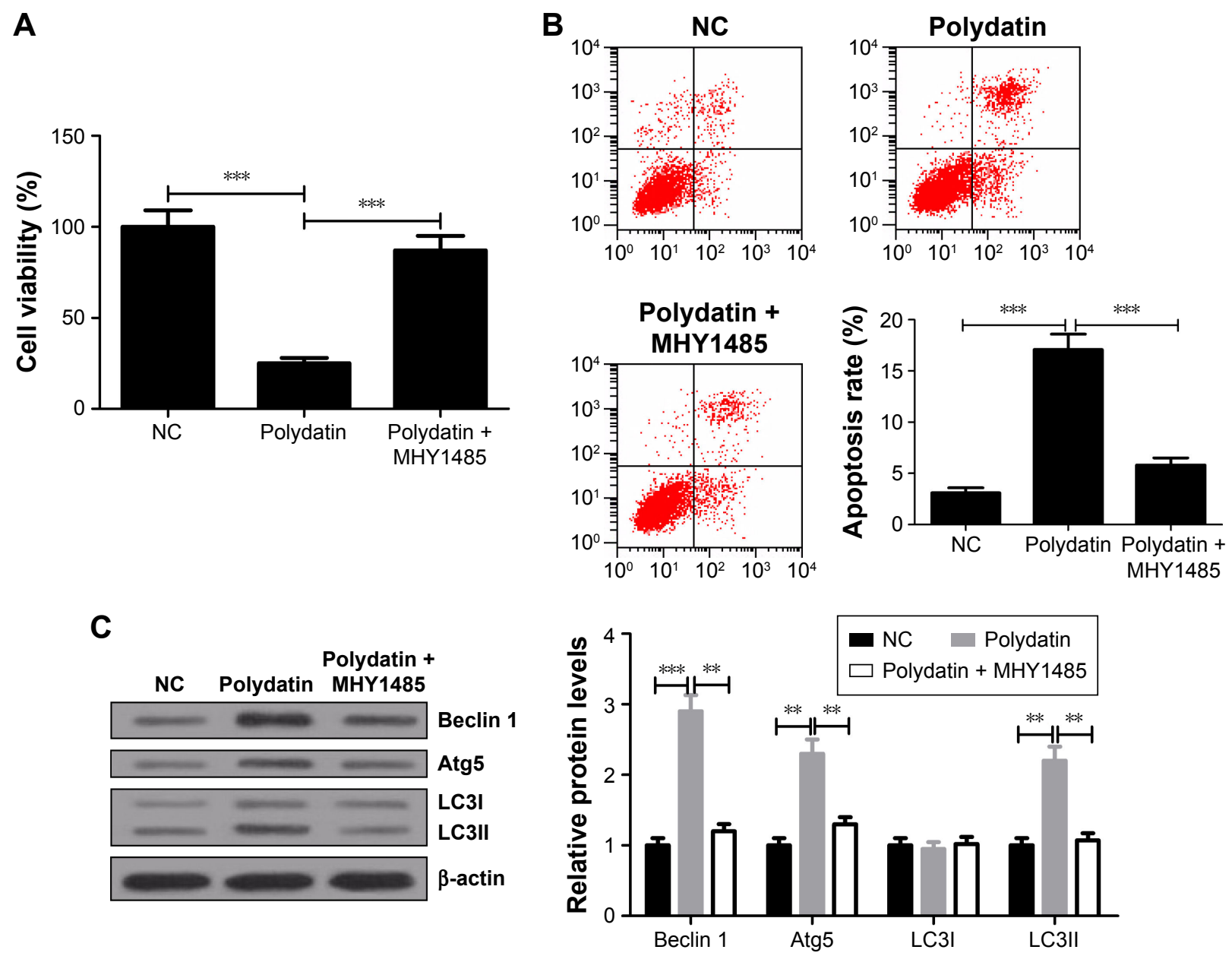

Figure 7 Viability suppression, apoptosis and autophagy induced by PD were repressed by MHYI485 in RPMI 8226 cells. Cells were treated with either $200 \mu$ mol/L PD or in combination with $10 \mu \mathrm{mol} / \mathrm{L}$ MHYI 485 for $24 \mathrm{~h}$. (A) Cell viability of RPMI 8226 cells was evaluated by CCK-8 assay. (B) Cell apoptosis in RPMI 8226 cells was examined by flow cytometry. (C) The levels of Beclin I, Atg5, LC3I and LC3II were determined by Western blot. $\beta$-actin was used as an internal control. Data are presented as mean \pm SD. $* * P<0.01$, *** P $<0.001$, compared to $\mathrm{NC}$.

Abbreviations: SD, standard deviation; PD, polydatin; CCK-8, cell counting kit8; NC, negative control.

Previous studies have found that PD played a potential role in inhibiting the proliferation of various tumors, such as in leukemia, breast cancer, lung cancer, cervical cancer and liver cancer ${ }^{12}$ and showed less toxicity to normal cells. However, little is known about the effect of PD on MM cells. Our results showed that PD functioned as a tumor suppressor in MM cells. The proliferation of MM cells decreased and apoptosis increased progressively along with the increasing concentrations of PD. In addition, it is indicated that cleaved caspase-3 and cleaved caspase-9 levels gradually increased along with the increasing concentrations of PD. Meanwhile, induction of apoptosis was accompanied by the downregulation of Bcl-2 and upregulation of Bax. That is, PD suppressed proliferation and promoted apoptosis of MM cells in timeand concentration-dependent manners. PD was previously regarded as a good therapeutic agent that induces apoptosis in cancer cells. ${ }^{21,22}$ Thus, PD may be used as a potent anticancer drug in MM therapy. A great many anticancer drugs inhibit tumor progression by inducing apoptosis. For example, in head and neck squamous carcinoma, dihydromyricetin increased the rate of apoptosis via the reactive oxygen species (ROS)signal transducer and activator of transcription-3 (STAT-3) signaling pathways. ${ }^{23}$ Cryptotanshinone induced apoptosis and prevented cell migration and invasion of melanoma cancer by ROS-mitochondrial apoptotic pathway and thus might be a potential anticancer drug for human melanoma treatment. ${ }^{24}$

Autophagy is a process by which cells degrade the damaged organelles and waste proteins..$^{25,26}$ Under stress situations, autophagy provides required nutrients to meet the needs of cellular metabolism and maintain a healthy cellular homeostasis and plays a crucial pro-survival role. ${ }^{27} \mathrm{Few}$ agents have been reported to induce apoptosis and activate autophagy. For example, Fan et al found that dihydromyricetin increased autophagy and apoptosis through the ROS-STAT3 signaling 
pathway in head and neck squamous carcinoma. ${ }^{23}$ Luo et al reported that quercetin nanoparticle promoted apoptosis and autophagy and suppressed cell growth by inhibiting JAK2 expression in cervical cancer. ${ }^{28}$ In this study, expression of autophagy-associated proteins was detected, such as Beclin 1, Atg5 (related to autophagosomes formation), LC3I (cytosolic form of key protein LC3 in autophagosome formation), LC3II (active membrane-bound form of LC3), HSP27 and HSP70. Western blot analysis showed that PD significantly augmented the levels of Beclin 1 and Atg5 in a concentration-dependent manner. PD treatment resulted in an increase in the ratio of LC3II/LC3I, generally accepted markers of autophagy, and a decrease in autophagy-associated proteins HSP27 and HSP70. Besides, this study has been found that the cell viability of RPMI 8226 cells, when treated with autophagy inhibitor 3-MA and PD, significantly increased compared with PD-treated group, whereas both apoptosis and autophagy induced by PD treatment obviously diminished when RPMI 8226 cells were incubated with 3-MA, which indicates that PD can also induce autophagy in addition to inhibiting apoptosis and proliferation in RPMI 8226 cells.

Therefore, this study has found that PD treatment significantly inhibited the phosphorylation of mTOR and p70s6k, which indicates that the mTOR/p70s6k signaling pathway might be a potential target pathway of PD. Previously, the $\mathrm{PI} 3 \mathrm{~K} / \mathrm{AKT} / \mathrm{mTOR} / \mathrm{p} 70$ s6 $\mathrm{k}$ signaling pathway has been found to be the target pathway of mollugin, gefitinib and pogostone inducing autopathy and apoptosis in glioblastoma cells, lung cancer and colorectal carcinoma. . 27,29,30 $^{2}$

The mTOR/p70s6k signaling pathway plays an important role in the regulation of autophagy, cell proliferation and cell survival in eukaryotic cells. ${ }^{31,32}$ In our study, the levels of autophagy-associated proteins, such as Beclin 1, Atg5, LC3I and LC3II, pro-apoptotic protein Bax, cleaved caspase-3, cleaved caspase-9 and the ratio of LC3II/LC3I, markedly increased after RPMI 8226 cells were treated with $\mathrm{mTOR} / \mathrm{p} 70 \mathrm{~s} 6 \mathrm{k}$ signaling pathway-specific inhibitor (rapamycin). Meanwhile, the anti-apoptotic protein Bcl-2 level was suppressed and cell viability dramatically decreased with rapamycin treatment. In addition, our results further implicated that activation of $\mathrm{mTOR} / \mathrm{p} 70 \mathrm{~s} 6 \mathrm{k}$ signaling pathway significantly eliminated the inhibitory effect of PD on proliferation and overturned PD-induced apoptosis and autophagy in RPMI 8226 cells. All these results demonstrated that inhibition of $\mathrm{mTOR} / \mathrm{p} 70 \mathrm{~s} 6 \mathrm{k}$ signaling pathway resulted in the inhibition of cell growth and induction of apoptosis and autophagy, while activation of this pathway showed the opposite effects, which is in agreement with the previous studies.

\section{Conclusion}

PD effectively suppressed cell growth and induced apoptosis and autophagy in MM cells through mTOR/p70s6k signaling pathway in vitro, which indicates that PD could be used as a potential anticancer drug for MM treatment. However, further research is needed to explore the anticancer effect of PD in vivo.

\section{Disclosure}

The authors report no conflict of interest in this work.

\section{References}

1. Stewart AK, Fonseca R. Prognostic and therapeutic significance of myeloma genetics and gene expression profiling. J Clin Oncol. 2005; 23(26):6339-6344.

2. Munshi NC, Anderson KC, Bergsagel PL, et al; International Myeloma Workshop Consensus Panel 2. Consensus recommendations for risk stratification in multiple myeloma: report of the International Myeloma Workshop Consensus Panel 2. Blood. 2011;117(18):4696-4700.

3. Cuendet M, Christov K, Lantvit DD, et al. Multiple myeloma regression mediated by bruceantin. Clin Cancer Res. 2004;10(3):1170-1179.

4. Sant M, Allemani C, Tereanu C, et al. Incidence of hematological malignancies in Europe by morphological subtype: results of the HAEMACARE project. Blood. 2010;116(19):3724-3734.

5. Matsumoto T, Jimi S, Migita K, Takamatsu Y, Hara S. Inhibition of glucose transporter 1 induces apoptosis and sensitizes multiple myeloma cells to conventional chemotherapeutic agents. Leuk Res. 2016;41: $103-110$.

6. Zhao KS, Jin C, Huang X, et al. The mechanism of polydatin in shock treatment. Clin Hemorheol Microcirc. 2003;29(3-4):211-217.

7. Zhao KS. Hemorheologic events in severe shock. Biorheology. 2005; 42(6):463-477.

8. Wang X, Song R, Chen Y, Zhao M, Zhao KS. Polydatin - a new mitochondria protector for acute severe hemorrhagic shock treatment Expert Opin Investig Drugs. 2013;22(2):169-179.

9. Cheng Y, Zhang HT, Sun L, et al. Involvement of cell adhesion molecules in polydatin protection of brain tissues from ischemia-reperfusion injury. Brain Res. 2006;1110(1):193-200.

10. Wang C, Luo Y, Lu J, Wang Y, Sheng G. Polydatin induces apoptosis and inhibits growth of acute monocytic leukemia cells. J Biochem Mol Toxicol. 2016;30(4):200-205.

11. Xu G, Kuang G, Jiang W, Jiang R, Jiang D. Polydatin promotes apoptosis through upregulation the ratio of $\mathrm{Bax} / \mathrm{Bcl}-2$ and inhibits proliferation by attenuating the $\beta$-catenin signaling in human osteosarcoma cells. Am J Transl Res. 2016;8(2):922-931.

12. Zhang Y, Zhuang Z, Meng Q, Jiao Y, Xu J, Fan S. Polydatin inhibits growth of lung cancer cells by inducing apoptosis and causing cell cycle arrest. Oncol Lett. 2014;7(1):295-301.

13. Strimpakos AS, Karapanagiotou EM, Saif MW, Syrigos KN. The role of mTOR in the management of solid tumors: an overview. Cancer Treat Rev. 2009;35(2):148-159.

14. Lee DF, Kuo HP, Chen CT, et al. IKK beta suppression of TSC1 links inflammation and tumor angiogenesis via the mTOR pathway. Cell.2007; 130(3):440-455.

15. Liu LZ, Zheng JZ, Wang XR, Jiang BH. Endothelial p70 S6 kinase 1 in regulating tumor angiogenesis. Cancer Res. 2008;68(19):8183-8188.

16. Levine B, Kroemer G. Autophagy in the pathogenesis of disease. Cell. 2008;132(1):27-42. 
17. Baldo P, Cecco S, Giacomin E, Lazzarini R, Ros B, Marastoni S. mTOR pathway and mTOR inhibitors as agents for cancer therapy. Curr Cancer Drug Targets. 2008;8(8):647-665.

18. Hay N. The Akt-mTOR tango and its relevance to cancer. Cancer Cell. 2005;8(3):179-183.

19. Inoki K, Corradetti MN, Guan KL. Dysregulation of the TSC-mTOR pathway in human disease. Nat Genet. 2005;37(1):19-24.

20. Pang X, Yi Z, Zhang J, et al. Celastrol suppresses angiogenesismediated tumor growth through inhibition of AKT/mammalian target of rapamycin pathway. Cancer Res. 2010;70(5):1951-1959.

21. Neto CC, Amoroso JW, Liberty AM. Anticancer activities of cranberry phytochemicals: an update. Mol Nutr Food Res. 2008;52(Suppl 1): S18-S27.

22. Kaur M, Agarwal R. Transcription factors: molecular targets for prostate cancer intervention by phytochemicals. Curr Cancer Drug Targets. 2007; 7(4):355-367.

23. Fan TF, Wu TF, Bu LL, et al. Dihydromyricetin promotes autophagy and apoptosis through ROS-STAT3 signaling in head and neck squamous cell carcinoma. Oncotarget. 2016;7(37):59691-59703.

24. Ye T, Zhu S, Zhu Y, et al. Cryptotanshinone induces melanoma cancer cells apoptosis via ROS-mitochondrial apoptotic pathway and impairs cell migration and invasion. Biomed Pharmacother. 2016;82:319-326.

25. Yue H, Li W, Liu P, Gao J, Miao J, Zhao J. Inhibition of autophagy promoted sphingosylphosphorylcholine induced cell death in non-small cell lung cancer cells. Biochem Biophys Res Commun. 2014;453(3): 502-507.
26. Komatsu M, Waguri S, Chiba T, et al. Loss of autophagy in the central nervous system causes neurodegeneration in mice. Nature. 2006; 441(7095):880-884.

27. Zhang L, Wang H, Zhu J, Xu J, Ding K. Mollugin induces tumor cell apoptosis and autophagy via the PI3K/AKT/mTOR/p70S6K and ERK signaling pathways. Biochem Biophys Res Commun. 2014;450(1): 247-254.

28. Luo CL, Liu YQ, Wang $P$, et al. The effect of quercetin nanoparticle on cervical cancer progression by inducing apoptosis, autophagy and antiproliferation via JAK2 suppression. Biomed Pharmacother. 2016;82: 595-605.

29. Zhao ZQ, Yu ZY, Li J, Ouyang XN. Gefitinib induces lung cancer cell autophagy and apoptosis via blockade of the PI3K/AKT/mTOR pathway. Oncol Lett. 2016;12(1):63-68.

30. Cao ZX, Yang YT, Yu S, et al. Pogostone induces autophagy and apoptosis involving PI3K/Akt/mTOR axis in human colorectal carcinoma HCT116 cells. J Ethnopharmacol. 2016;pii: S0378-S8741(16): 30453-30456.

31. Borders EB, Bivona C, Medina PJ. Mammalian target of rapamycin: biological function and target for novel anticancer agents. Am J Health Syst Pharm. 2010;67(24):2095-2106.

32. Yap TA, Garrett MD, Walton MI, Raynaud F, de Bono JS, Workman P. Targeting the PI3K-AKT-mTOR pathway: progress, pitfalls, and promises. Curr Opin Pharmacol. 2008;8(4):393-412.
OncoTargets and Therapy

\section{Publish your work in this journal}

OncoTargets and Therapy is an international, peer-reviewed, open access journal focusing on the pathological basis of all cancers, potential targets for therapy and treatment protocols employed to improve the management of cancer patients. The journal also focuses on the impact of management programs and new therapeutic agents and protocols on

\section{Dovepress}

patient perspectives such as quality of life, adherence and satisfaction The manuscript management system is completely online and includes a very quick and fair peer-review system, which is all easy to use. Visit http://www.dovepress.com/testimonials.php to read real quotes from published authors. 